\title{
Editorial
}

CYTOLOGICA

Published online: April 27, 2011

DOI: $10.1159 / 000324733$

\section{Transforming Cytology}

James Cameron's Avatar envisioned a digital new world of imagination afire with images of technologic progress in conflict with the culture of a complacent, naïve society. Avatar (generically) invokes the image of embodiment as a 'new personification of a familiar idea'. It is both representational and animated, an action effigy figure imbued with the motivational freedom of the alter ego. As a graphics-based interactive change agent it is, by some stretch, a motivational manifestation of an identity that transforms to survive. Avatar could be the icon of the cytologist on the brink of change to avoid species extinction in a universe bombarded by meteoric technological advancements and deep roots 'in the way we've always done it'.

There are forces influencing the evolution of cytology: automation and digital imaging, molecular testing for HPV and genomic aberrations, vaccines, workforce issues, the decline and closure of cytology schools with dismal enrollment in surviving programs, negative attitudes, legislative restrictions on scope of practice, a failing economy with declining reimbursement, the diagnostic challenge to do more with less, the impact of health consumerism on the laboratory industry, globalization of pathology services, digital imaging, telemedicine, and a frightening physician shortage with critical international pathologist shortage. Patients are more proactive in their own care, employer wellness programs promote consumerism, a rise in self-testing products diminishes lab use and centralization of laboratories changes employment dynamics.

These collective forces and the advent of molecular testing for HPV and its impact on cytotechnology have produced speculation about the viability/demise of the Pap test and job security for cytotechnologists. Cytopa- thologists have been redirected from laboratory to patient by demands for ultrasound and other image-guided fine needle aspiration biopsies with more sophisticated analyses by molecular methods and immunostains creating a need for professional support. In the middle of the last decade, the executive board of the American Society of Cytopathology (ASC) was challenged to initiate a formal redefinition of scope of practice for cytotechnologists that would embrace new technologies and the relationship to fine needle aspiration and other facets of health care delivery. The Forbes Group was engaged for this purpose and their report, Plotting the Future of Cytotechnology: An Environmental Analysis of the Driving Forces of Cytology, was published in June 2007. The report exceeded anticipated responses by characterizing a global snapshot of where medicine is today, how it is changing, and what cytology must do to retain a preeminent position in diagnosis, therapy and prognosis. The challenge of how to enhance scope of practice for cytotechnologists became the larger issue of how pathology as the parent discipline and cytopathology as its derivative must change in a changing world. A cell-inspired Avatar was needed to embody a new personification of a familiar idea that transforms to survive.

Forbes reflected that market dynamics exist to justify a new, or at least transformed profession. Inherent is the breakdown of the traditional linear care delivery model (patient-clinician-pathologist-technologist) as new relationships emerge. The most important new relationship is that of the pathologist with the patient. As pathology becomes more interventional (ultrasound-guided biopsies by cytopathologists), new roles are defined at the patient interface. The predicted track is that pathologists will be transformed from clinicians' consultants to clinical pro-

\section{KARGER}

Fax +4161306 1234

E-Mail karger@karger.ch

www.karger.com
(C) 2011 S. Karger AG, Basel

$0001-5547 / 11 / 0553-0225 \$ 38.00 / 0$

Accessible online at:

www.karger.com/acy
Correspondence to: Dr. David B. Kaminsky

Palm Springs Pathology Services

Suite 203, 1401 North Palm Canyon Drive

Palm Springs, CA 92262 (USA)

Tel. +1 760327 6777, E-Mail dkaminsky@pspathology.com 
viders and that cytotechnologists will be liberated to a more flexible scope of practice as extenders of the more traditional pathologists' tasks. The Avatar of the advanced cytology practitioner is still on the graphics table, and its design will either explode with imagination or be sabotaged and subdued by the hangers-on of tradition and territorial domain.

If we as cytology professionals are to access a digital new world of imagination afire with images of technologic progress, we must commit to a process that achieves an evolved pathologist, a transformative Avatar that supersedes its traditional laboratory-based version. The portrait of the evolved pathologist is vibrant with possibilities: the evolved pathologist transitions focus from laboratory to patient and establishes a strong patient interface through interventional procedures (ultrasoundguided fine needle aspiration biopsies), consultative services and direct patient education. The evolved pathologist takes leadership responsibilities in adapting new technologies to direct patient care through targeted therapies, prognostic indicators and genetic assessments. Doing more with less is a reality in the new world, and it should be the pathologist who orchestrates the translation of cell secrets to desired patient outcomes. The evolved pathologist embraces the fusion of specialized disciplines and through the dissolution of boundaries and barriers creates a medical team with combined expertise that places the patient at center. The evolved pathologist shifts focus from the diagnostic sample to the complete patient: physical examination and history taking preparatory to performance of the fine needle aspira- tion biopsy, preoperative physical examination of patients scheduled for frozen sections and face-to-face consultations ensure the direct contact of the pathologist with the patient, open communication and education and give identity to one of the most important diagnostic resources in medical practice, the patient-focused pathologist. The evolved pathologist enhances his/her expertise in digital imaging, archiving and telemedicine to facilitate exchange of information with evidence-based data. This endeavor leads naturally to the integrated report which incorporates images of the needle in its target, the endoscopic appearance of gastrointestinal lesions, the clinical presentation of grossly visible lesions, and graphic illustrations of procedures that resulted in diagnostic samples. The evolved pathologist formats his integrated report to include patient education with color photographs and graphics that clinicians can utilize to present diagnoses and illustrate the disease. The report is an interface with clinical colleagues and patients and should be formulated and valued as an important communication and educational resource. Report and rapport are buzzwords that underscore these ideas. The evolved pathologist has discovered the artist inside the physician and utilizes esthetic options as conduits to compassionate care (art therapy, report design, facility ambiance).

The conversion or transformation to the patient-centered pathology practice in which there is direct patient interface reminds the pathologist that he or she is a physician and reinforces an invigorated and self-satisfying role liberated to evolve and survive.

David B. Kaminsky, Palm Springs, Calif., USA 